\title{
Fast Multipole Method in Layered Media: 2-D Electromagnetic Scattering Problems ${ }^{\dagger}$
}

\author{
Levent Gürel* and M. I. Aksun \\ Dept. of Electrical and Electronics Engineering \\ Bilkent University \\ Bilkent, Ankara, Turkey
}

\section{Introduction}

The formulation of layered-media problems have traditionally been carried out in the spectral domain due to the availability of the Green's functions in closed forms [1,2]. Recently, a series of techniques have been developed to obtain closed-form Green's functions (CFGFs) for layered media in the spatial domain [3,4]. The use of the CFGFs in a method-of-moments (MOM) formulation reduces the matrix-filling time by several orders of magnitude compared to the spectral-domain formulation. However, it, does not reduce the computational complexities of the matrix-filling time and the memory requirement, which are both $O\left(N^{2}\right)$. Most importantly, despite the great savings in the matrix-filling time, the solution of the $N \times N$ dense matrix equation remains, which requires $O\left(N^{3}\right)$ operations in a direct scheme or $O\left(N^{2}\right)$ operations per iteration in an iterative scheme.

On another front, several researchers are working to reduce the computational complexities and the memory requirements of the solution of integral equationis of electromagnetics. For the iterative solutions of the integral equations based on the Helmhoitz equation, the fast multipole method (FMM). which has $O\left(N^{3 / 2}\right)$ complexity per iteration, has recently attracted attention $[5,6]$. The FMM employs a haumonic expansion of the closed-form Green's function and has been limited to the homogeneous-medium problems so far. By obtaining a closed-form expression for the spatial-domain Green's function for an arbitrarily layered medium and by interpreting each term of the expression as a discrete complex image, we have been able to apply the FMM to the solution of layered-media problems. Thus, we have obtained a fast solution technique for the layeredmedia problems and extended the applicability of the FMM from homogeneous-medium problems to layered-medium problems.

\section{Fast Multipole Method in a Layered Medium}

The $y$-directed electric field at point $\rho=\hat{x} x+\hat{z} z$ due to a $y$-directed line source with unit amplitude located at point $\rho^{\prime}=\hat{x} x^{i}+\hat{z} z^{\prime}$ is given by

$$
G\left(\rho, \rho^{\prime}\right)=-\frac{\omega \mu}{4 \pi} \int_{-\infty}^{\infty} d k_{x} e^{i k_{x}\left(x-x^{\prime}\right)} \frac{1}{k_{z}}\left[e^{i k_{z}\left|z-x^{\prime}\right|}+\tilde{R}^{T E} e^{i k_{z}\left(z+z^{\prime}\right)}\right]
$$

'This work was supportcd in part by NATO's Scientific Affairs Division in the framework of the Science for Stability Programme and in part by the Scientific and Technical Research Council of Turkey (TUBITAK) under contracts EEEAG-132 and EEEAG-163.

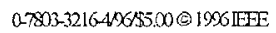


where $\tilde{R}^{T E}$ is the generalized reflection coefficient defined at the $z=0$ plane due to an arbitrarily layered substrate below this plane.

Using a robust technique by Aksur [4], Eq. (1) can be converted to a closed-form expression given by

$$
G\left(\boldsymbol{\rho}, \boldsymbol{\rho}^{\prime}\right)=-\frac{\omega \mu}{4}\left[H_{0}^{(1)}\left(k\left|\rho-\boldsymbol{\rho}^{\prime}\right|\right)+\sum_{n=1}^{N_{t}} a_{n} H_{0}^{(1)}\left(k\left|\boldsymbol{\rho}-\boldsymbol{\rho}_{n}^{\prime}\right|\right)\right]
$$

where $\left|\rho-\rho_{n}^{\prime}\right|=\sqrt{\left(x-x^{\prime}\right)^{2}+\left(z+z^{\prime}+i \alpha_{n}\right)^{2}}$ and $a_{n}$ and $\alpha_{n}$ are complex constants for $n=1,2, \ldots, N_{I}$.

The FMM is based on the expansion of the Green's function using the addition theorems and no such expansions exist for the layered-media Green's functions given in Eqs. (1) and (2). However, we can still employ the FMM in the solution of a layeredmedium problem if we interpret Eq. (2) as the linear superposition of the field due to a source at $\rho^{\prime}$ and the fields due to $N_{I}$ discrete complex images (DCIs) located at $\rho_{n}^{\prime}=\hat{x} x^{\prime}+\hat{z}\left(-z^{\prime}-i \alpha_{n}\right)$. The DCIs are located at complex coordinates and, therefore, we need to use addition theorems for wave functions with complex arguments.

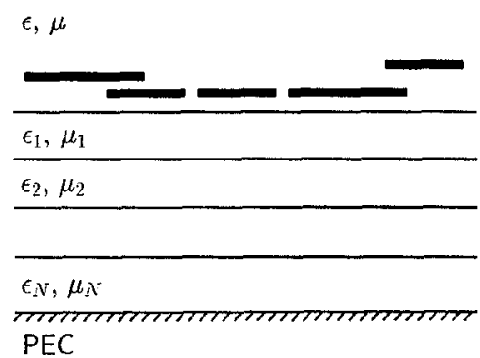

(a)

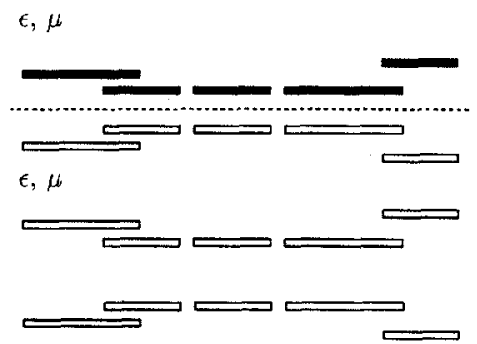

(b)

Fig. 1. (a) Original problem in a layered medium. (b) Equivalent problem with DCIs in a homogeneous medium.

With the DCI interpretation, an equivalent problem is set up in a homogeneous mcdium. In this equivalent problem, which is illustrated in Fig. 1 (b), $N_{I}$ image sources in a homogeneous medium are defined corrcsponding to each original source in the layeredmedium problem [Fig. 1(a)]. 'L'hus, if $N$ testing functions are defined on the original conductors, $N\left(N_{I}+1\right)$ basis functions are defined on the original conductors and their images.

The computation of the fields of $N\left(N_{I}+1\right)$ basis functions on the $N$ testing functions is carried out using the FMM and repeated several times in an iterative scheme. Since $N_{I}$ is a constant, this specific implementation of the FMM for layered-media problems has $O\left(N^{3 / 2}\right)$ computational complexity per iteration and $O\left(N^{3 / 2}\right)$ memory requircment as its homogeneous-medium counterpart. 
$\epsilon_{0}, \mu_{0}$

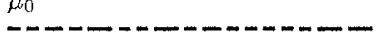

(a)

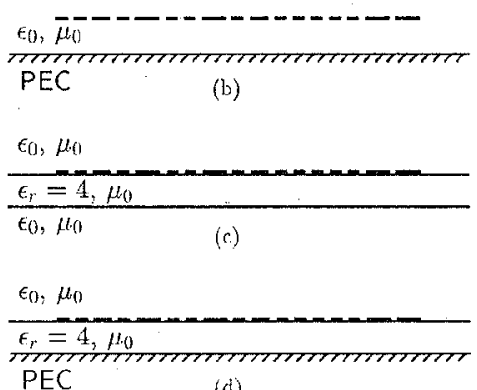

PEC (d)

Fig. 2. Examples of layered structures.

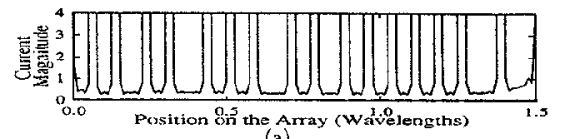

(a)

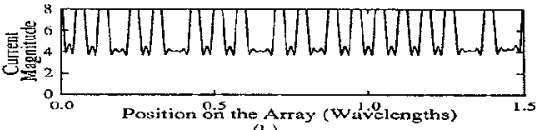

(b)

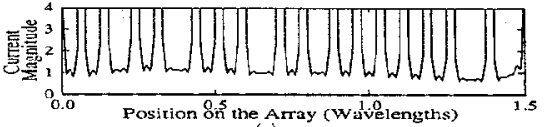

(c)

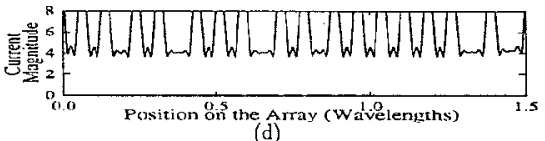

(d)

Fig. 3. Current distributions on the array corresponding to the example structures of Fig. (2).

\section{Computational Results}

In orcler to demonstrate the accuracy and the efficiency of the layered-media implemen1ation of the FMM, a series of structures (as illustrated in Fig. 2), to which the method can be applied, have been designed. Common to all these structures is an irregular, finite and planar array of strips, which has an overall extent of $1.5 \lambda_{0}$, as shown in Fig. 2(a). The same array is placed $\lambda_{0} / 100$ away from a conducting plane in Fig. $2(b)$ and the thickness of the dielectric slab in Figs. $2(\mathrm{c})$ and (d) is also $\lambda_{0} / 100$. Figures 3(a)-(d) present the plots of the magnitude of the current on the arrays of Figs. 2(a)-(d), respectively, when illuminated by parallel-polarized plane waves incident at $45^{\circ}$. These results are also checked against results obtained by employing direct solution with Gainssian elimination and iterative solution with ordinary matrix-vector multiplication. All three solution techniques are found to generate results that agree with each other for several digits, testifying to the accuracy of the iterative solution with layered-medium implementation of the FMM.

Figure 4(a) compares the solution times of the layered-medium implementation of the FMM with the Gaussian elimination as the array geometry of Fig. 2(c) becomes larger. Similarly, Fig. 4(b) compares the per-iteration solution times of the layered-medium implementation of the FMM with the ordinary matrix-vector multiplication. As for the filling time, since only a sparse matrix of the near-feld interactions is filled in the FMM as opposed to filling an $N \times N$ dense matrix in the direct solution, filling time of the FMM is always lower. 


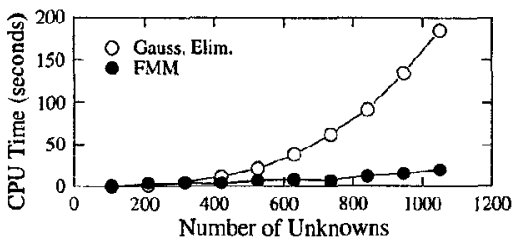

(a)

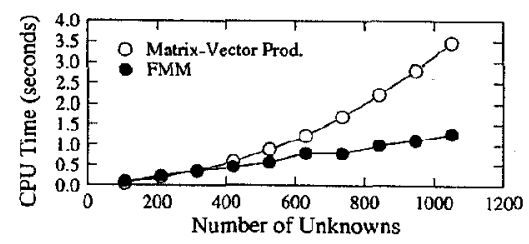

(b)

Fig. 4. (a) Comparison of the solution times of the layered-medium implementation of the FMM and the Gaussian elimination. (b) Comparison of the per-iteration solution times of the layered-medium implementation of the FMM and the ordinary matrix-vector multiplication.

\section{Conclusions}

The applicability of the FMM has been extended to layered-media problems. As an example, we have demonstrated the solution of the scalar Helmholtz equation for the electromagnetic scattering from a two-dimensional planar array of horizontal strips on a layered substrate.

\section{References}

[1] T. Itoh, "Spectral domain immittance approach for dispersion characteristics of generalized transmission lines," IEEE Trans. Microwave Theory Tech., vol. MTT28, pp. 733-736, July 1980.

[2] W. C. Chew and L. Gürel, "Reflection and transmission operators for strips or disks embedded in homogeneous and layered media," IEEE Trans. Microwave Theory Tech., vol. MTT-36, pp. 1488-1497, Nov. 1988.

[3] Y. L. Chow, J. J. Yang, D. F. Fang, and G. E. Howard, "A closed-form spatial Green's function for the thick microstrip substrate," IEEE Trans. Microwave Theory Tech., vol. MTT-39, pp. 588-592, Mar. 1991.

[4] M. I. Aksun, "A robust approach for the derivation of closed-form Green's func tions," IEEE Trans. Microwave Theory Tech., in press.

[5] V. Rokhlin, "Rapid solution of integral equations of scattering theory in two dimen sions," J. Comput. Phys., vol. 86, pp. 414-439, Feb. 1990

[6] C. C. Lu and W. C. Chew, "Fast algorithm for solving hybrid integral equations," Proc. IEE, vol. 140, Part H, pp. 455-460, Dec. 1993. 